\title{
Comprehensive Metabolome and Volatilome Analyses in Eggplant and Tomato Reveal Their Differential Responses to Tuta absoluta Infestation
}

OPEN ACCESS

Edited by:

Shahid Siddique,

University of California, Davis,

United States

Reviewed by:

Samir Jaoua,

Qatar University, Qatar

Frank Chidawanyika,

International Center of Insect

Physiology and Ecology (ICIPE), Kenya

*Correspondence:

Yaobin Lu

luybcn@163.com

Youming Hou

ymhou@fafu.edu.cn

${ }^{\dagger}$ These authors have contributed equally to this work

Specialty section:

This article was submitted to Plant Pathogen Interactions,

a section of the journal

Frontiers in Plant Science

Received: 11 August 2021 Accepted: 14 September 2021 Published: 03 November 2021

Citation:

Chen L, Li X, Zhang J, He T, Huang J, Zhang Z, Wang Y, Hafeez M, Zhou S,

Ren X, Hou Y and Lu Y (2021)

Comprehensive Metabolome and Volatilome Analyses in Eggplant and Tomato Reveal Their Differential

Responses to Tuta absoluta Infestation.

Front. Plant Sci. 12:757230

doi: 10.3389/fp/s.2021.757230

\begin{abstract}
Limin Chen ${ }^{1,2,3+}$, Xiaowei $\mathrm{Li}^{1+}$, Jinming Zhang ${ }^{1}$, Tianjun He ${ }^{3}$, Jun Huang ${ }^{1}$, Zhijun Zhang ${ }^{1}$, Yeyang Wang ${ }^{3}$, Muhammad Hafeez ${ }^{1}$, Shuxing Zhou ${ }^{1}$, Xiaoyun Ren ${ }^{1}$, Youming Hou ${ }^{2 *}$ and Yaobin $\mathrm{Lu}^{1 *}$

'State Key Laboratory for Managing Biotic and Chemical Threats to the Quality and Safety of Agro-Products, Institute of Plant Protection and Microbiology, Zhejiang Academy of Agricultural Sciences, Hangzhou, China, ${ }^{2}$ State Key Laboratory of Ecological Pest Control for Fujian and Taiwan Crops, Key Lab of Biopesticide and Chemical Biology, Ministry of Education \& Fujian Key Laboratory of Insect Ecology, College of Plant Protection, Fujian Agriculture and Forestry University, Fuzhou, China, ${ }^{3}$ Integrated Plant Protection Center, Lishui Academy of Agricultural and Forestry Sciences, Lishui, China
\end{abstract}

The South American tomato pinworm, Tuta absoluta, is one of the most destructive insect pests in Solanaceae crops, particularly in tomatoes. Current methods of management have proven somewhat effective but still require a more efficacious management strategy to limit its havoc on crop yield. Tomato is much more predisposed to $T$. absoluta as compared with other plants such as eggplants, but the underlying causes have not been fully determined. We conducted this study to unravel the volatile organic compounds (VOCs) and primary/secondary metabolites that account for the differential response of tomatoes and eggplants to T. absoluta infestation. We performed widely targeted comparative metabolome and volatilome profiling by ultraperformance liquid chromatography-tandem mass spectrometry (UPLC-MS/MS) and headspace solid-phase microextraction coupled to gas chromatography-mass spectrometry (HS-SPME/GC-MS), respectively, on eggplants and tomatoes under control and T. absoluta infestation conditions. Overall, 141 VOCs and 797 primary/secondary metabolites were identified, largely dominated by aldehyde, alcohols, alkanes, amine, aromatics, a heterocyclic compound, ketone, olefin, phenol, and terpenes. Most of the VOCs and primary/secondary metabolites from the terpene class were largely differentially regulated in eggplants compared with tomatoes. Eggplants emitted several compounds that were lower or completely absent in tomatoes either under control conditions or after T. absoluta infestation. The results from an electroantennogram showed that 35 differentially accumulated VOCs could elicit female T. absoluta response, implying that these volatile compounds significantly alter the behavior of this pest. These findings demonstrated that 
differentially accumulated metabolites and volatile compounds play major roles in eggplant resistance to $T$. absoluta infestation as these compounds were regulated upon attack by $T$. absoluta. Our findings can assist in integrated pest management efforts by developing appropriate control measures against $T$. absoluta in Solanaceae production.

Keywords: electroantennography, biocontrol approach, host preference, plant defense metabolites, volatiles

\section{INTRODUCTION}

The South American tomato pinworm [Tuta absoluta (Meyrick)] has become one of the most destructive pests in tomato production worldwide (Bawin et al., 2016; Zhang et al., 2020). Tuta absoluta belongs to the Gelechiidae family in the order Lepidoptera (Zhang et al., 2020) and was first described in South America in 1917, which caused extensive damage to tomatoes and other plants (Biondi et al., 2018; Cherif and Verheggen, 2019; Tarusikirwa et al., 2020). The pest has since spread widely to $\sim 110$ countries in Africa, America, Asia, Europe, and the Middle East through the large-scale importation of tomatoes in those countries (Desneux et al., 2010; Biondi et al., 2018; Mansour et al., 2018; Guimapi et al., 2020). Tuta absoluta feeds, overwinters, and breeds on a broad range of hosts such as eggplant, potato, nightshade, tomato, and tobacco (Silva et al., 2021). Tomato is reported to be the most preferred host (Shah et al., 2015; Negi et al., 2018). The larvae damage the leaves, buds, stems, and fruits, with a detrimental effect on fruit yield and quality (Torres et al., 2001; Desneux et al., 2010). The economic effect of this pest in tomato production and yield cannot be overemphasized (Campos et al., 2017). The poor management of T. absoluta infestations can lead to $80-100 \%$ crop loss (Maluf et al., 2010).

Solanaceae vegetables, such as tomatoes (Solanum lycopersicum L.) and eggplants (Solanum melongena L.) are multi-nutritive vegetables that are cultivated all over the world and consumed in both fresh and processed forms (Kavanaugh et al., 2007; Gerszberg et al., 2015). However, biotic factors such as insects and pathogens are significant impediments to their long-term development (Shah et al., 2015). Tomato and eggplant cultivation under field and greenhouse conditions are rapidly expanding into new regions in China (Desneux et al., 2010; Biondi et al., 2018; Cherif and Verheggen, 2019; Zhang et al., 2020). Although researchers and the government have attempted to limit the spread of $T$. absoluta in China by working together, the pest was found in the Xinjiang region of China in 2017 (Zhang et al., 2020). In 2018, the damage of this pest has also been reported in protected-field fresh marketing tomatoes in Lincang, Yunnan province (Cherif and Verheggen, 2019; Zhang et al., 2020). Recent studies have found that $T$. absoluta is spreading at $800 \mathrm{~km}$ each year (Cherif and Verheggen, 2019; Li Xiaowei,

Abbreviations: ANOVA, analysis of variance; DAVOC, differentially accumulated volatile organic compounds; EAG, electroantennogram; GCMS, gas chromatography-mass spectrometry; PCA, Principal component analysis; PLS-DA, partial least squares discriminant analysis; QC, quality control; UPLCMS/MS, ultra-performance liquid chromatography-tandem mass spectrometry; VIP, variable importance in projection; VOC, volatile organic compounds.
2019). In the light of these new findings, specifically in terms of migratory patterns, a more focused tracking of tomatogrowing areas is necessary throughout China; otherwise, tomatoes elsewhere in the country will lose their resistance to the pest.

There have been several efforts to monitor the global invasions of T. absoluta over the past decade (Campos et al., 2017; Han et al., 2018; Li et al., 2021). Chemical control is the primary measure to manage T. absoluta in both native and invaded areas (Silva et al., 2018; Langa et al., 2021). However, the large use of chemical pesticides led to environmental pollution and food safety challenges (Machekano et al., 2018). Consequently, various pesticide-resistant strains have emerged in different locations (Zappal et al., 2013; El-Arnaouty et al., 2014; Biondi et al., 2015). Alternative methods of management, such as using its natural enemies (El-Arnaouty et al., 2014; Biondi et al., 2015; Silva et al., 2018; Langa et al., 2021) and pheromones, have proven somewhat effective in T. absoluta population suppression (Aksoy and Kovanci, 2016; Mirhosseini et al., 2019), but still requires a more efficacious management strategy to limit its havoc on crop yield.

Recent advances have been made in tomatoes through the use of genetic and genomic techniques to limit insect damages (Comparative biochemical and transcriptome analyses in tomato and eggplant reveal their differential responses to Tuta absoluta infestation | Mendeley; Cocco et al., 2013; Zhou et al., 2020; Chen et al., 2021). However, current research on the South American tomato pinworm mainly focuses on invasion status, host range, pesticide resistance, and natural enemy control. There are relatively limited research reports on the metabolites induced or repressed by $T$. absoluta infestation and on host preference and interaction mechanism at the molecular level (Rostami et al., 2020). The survival and feeding preference of herbivorous insects are partly controlled by plant metabolites. An attack or wounding by $T$. absoluta larvae often induces specific metabolic variations in plants to regulate feeding, mating, and oviposition (Cha et al., 2008; Gripenberg et al., 2010; Fatouros et al., 2016). A study on tomatoes revealed that the interaction between brassinosteroids and jasmonates is needed to counteract attacks by T. absoluta (Campos et al., 2009). Phytohormones such as auxin, brassinosteroids, ethylene, gibberellin, jasmonates, and salicylic acid are reported to act synergistically or antagonistically to activate signal transduction upon insect feeding or wounding (Howe and Jander, 2008; War et al., 2011). Besides the effects of plant metabolites on survival and feeding preference, the preference of herbivorous insects for host plants mainly depends on the choice of adult oviposition and the suitability of larvae on host plants (War et al., 2011). Plant volatile organic compounds (VOCs) do 

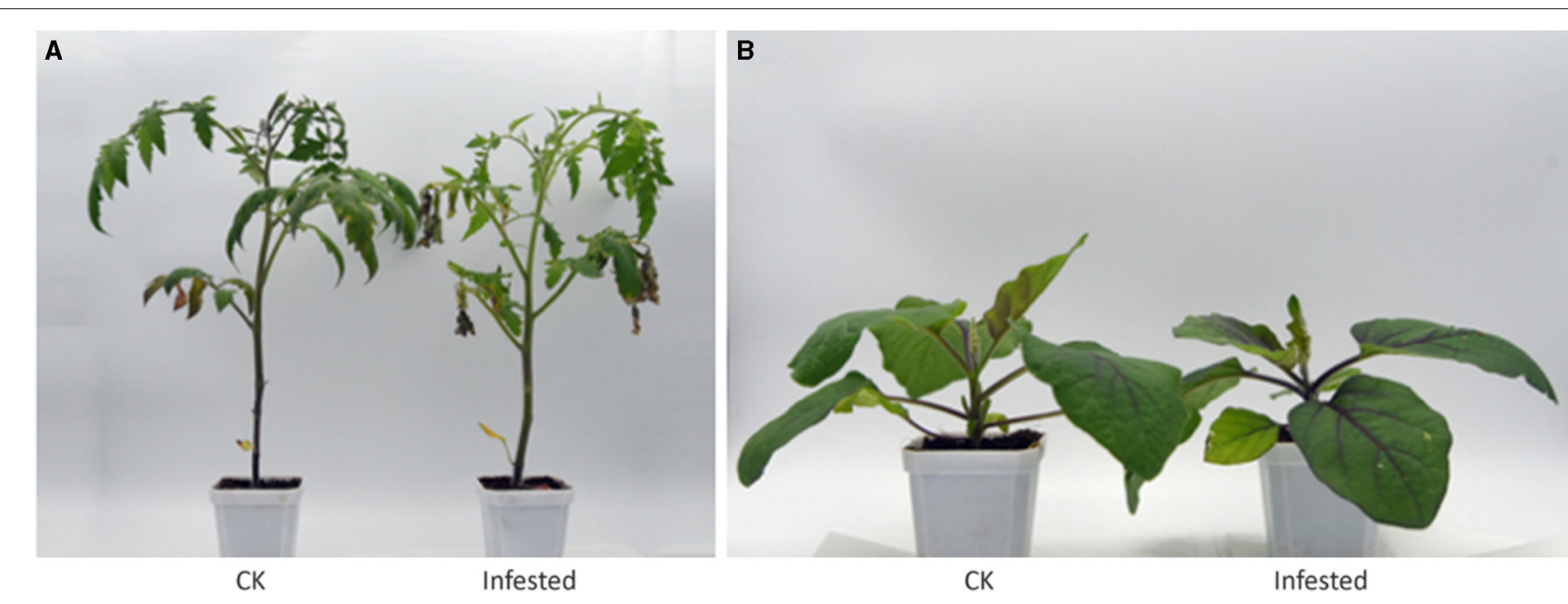

FIGURE 1 | Healthy and infested plants of tomato (A) and eggplant (B). The infested plants were obtained in a growth chamber $48 \mathrm{~h}$ post-T. absoluta infestation. CK, control plant.

not only play an important role in the interaction between plants and insects but are important chemo-information for phytophagous insects to locate host plants (Proffit et al., 2011; De Backer et al., 2015; Anastasaki et al., 2018). The role of plant volatiles in integrated pest management has increasingly gained research attention (Anastasaki et al., 2018). After a tomato is infested by South American tomato pinworm larvae, the plant can trigger herbivore-induced plant volatiles (HIPVs) by releasing (z)-3-hexen-1-ol, methyl salicylate, and indole-3 acetic acid to halt further egg-laying (Leitner et al., 2005). Similarly, in related studies on oviposition-inducing volatiles (OIPVs), it was found that the oviposition behavior of $T$. absoluta adults can induce the production of the tomato volatiles (z)-3-hexen-1-ol. In a study on the interaction between two natural enemies of $T$. absoluta, it was found that tomato plants with natural enemies can release the volatile octyl acetate to attract the egg-laying larvae of $T$. absoluta adults (Howe and Jander, 2008). Again, studies have shown that the compositions of different tomato volatiles are also different, and there are certain differences in the attracting ability of tomato leaf miners (War et al., 2011; Rostami et al., 2020).

Researchers have been investigating the various mechanisms of $T$. absoluta infestations in tomatoes and other species (Desneux et al., 2010; Cherif and Verheggen, 2019; Silva et al., 2021). In our earlier studies, the T. absoluta growth and survival rate in tomatoes was found to be substantially higher than in eggplants (Li Xiaowei, 2019). The mechanism that causes T. absoluta to prefer tomatoes over eggplants has been partly attributed to the differences in the behavioral selection and fitness of different host plants by herbivorous insects (Sankarganesh et al., 2017). Thus, the females of herbivorous insects lay more eggs on host plants that are conducive to the growth and development of their offspring, making the offspring more attracted to the host plants (Arnó et al., 2019). These differences in the behavioral selection and fitness of herbivorous insects to host plants mainly depend on the differential production of metabolites and volatiles by different host plants (Sylla et al., 2019). Some herbivorous insects can secrete corresponding detoxification enzymes for the degradation of toxic and harmful substances produced by host plants (Cherif and Verheggen, 2019). The current study profiled the metabolites and volatiles induced in tomatoes and eggplants in response to T. absoluta infestation. We also profiled the key plant metabolites that cause differences in the behavioral selection and fitness of $T$. absoluta and assessed the interaction between T. absoluta in tomatoes and eggplants and their effect on the synthesis of key plant metabolites. Theoretically, the study provided insights into host preferences by $T$. absoluta, which enriches our understanding of T. absoluta invasion biology and, in practice, as a guide for the development and application of plant-derived attractants and repellents against $T$. absoluta.

\section{RESULTS}

\section{Targeted Volatilome and Metabolome Profiling of Healthy and T. absoluta Infested Leaf Samples in Tomatoes and Eggplants}

This study was undertaken to identify the metabolites and volatiles that may account for the T. absoluta preference for tomatoes than eggplants as observed in a previous study (Li et al., 2019). The two species of Solanaceae were inoculated with T. absoluta in a growth chamber (Figure 1). Tomatoes exhibited severe symptoms of leaf yellowing and other symptoms associated with $T$. absoluta infestation (Mutamiswa et al., 2017). The majority of the fallen T. absoluta mature larvae were observed under the tomato plants, an 


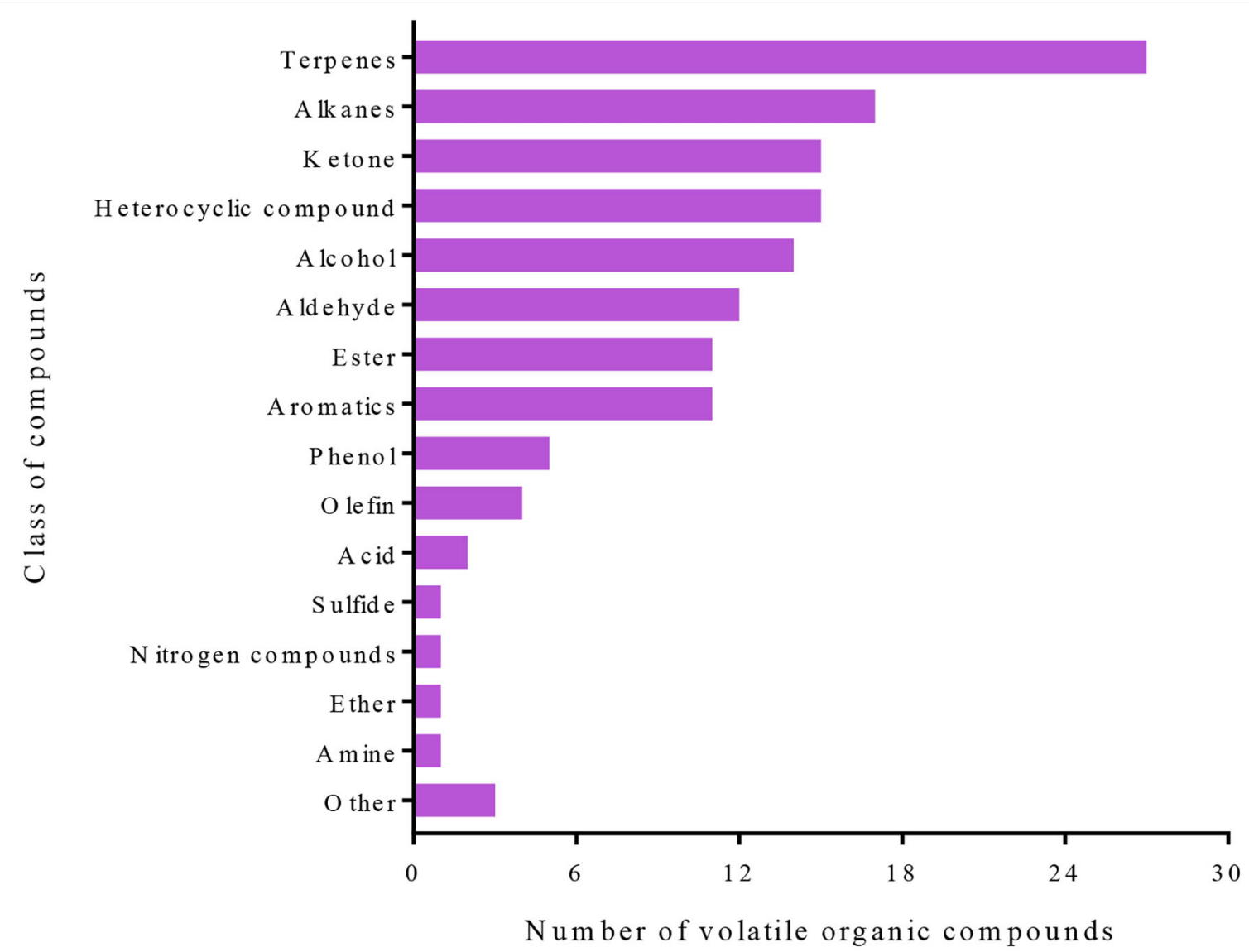

FIGURE 2 | Classes of volatile organic compounds identified in the control and infested leaves of tomatoes and eggplants.

indication that $T$. absoluta thrived and multiplied more easily in tomatoes than in eggplants. We sampled the two species under control conditions (healthy leaf free from $T$. absoluta infestation) and T. absoluta-infested conditions to quantitatively profile VOCs and primary/secondary metabolites with headspace solid-phase microextraction (HS-SPME) coupled with gas chromatography-mass spectrometry (GC-MS) and ultra-performance liquid chromatography-tandem mass spectrometry (UPLC-MS/MS).

This study has identified 141 VOCs, which were predominantly from terpenes, alkanes, ketone, heterocyclic compounds, alcohol, aldehyde, aromatics, phenol, and olefin classes that were detected in the four groups that had been evaluated (Figure 2). A hierarchical clustering analysis based on the ion intensities of the VOCs detected in the four groups (Tomato-Control, Tomato-T. absoluta infested, Eggplant-Control, and Eggplant-T. absoluta infested) clustered them into two main VOC clusters (Figure 3). Each cluster consisted of only one species of Solanaceae with its biological repeats (either control or T. absoluta infested) sub-clustered together. The results indicated that there are significant differences in the volatile profiles between tomatoes and eggplants either in control conditions or upon infestation by T. absoluta.

A total of 797 primary/secondary metabolites were identified among the four groups. These metabolites were from flavonoids, alkaloids, phenolic acids, lipids, amino acids and derivatives, organic acids, nucleotides and derivatives, lignans and coumarins, terpenoids, tannins, steroids, and others (Figure 4). Interestingly, one steroid compound (Aculeatiside A) was detected only in eggplants under the two conditions. We subjected the metabolite ion intensities of the four groups to hierarchical clustering analysis, resulting in two main clusters (Figure 5). Cluster I comprised solely of tomatoes and cluster II consisted of samples from eggplants (Figure 5). The three biological replicates of tomatoes or eggplants formed one sub-cluster in each cluster. This trend indicated that a limited number of primary/secondary compounds were induced by $T$. absoluta infestation. The clustering patterns of the metabolomes and volatilomes of biological repeats in the same sub-cluster pinpointed the nature of compounds and the repeatability of compounds in tomato and eggplant under either control or $T$. absoluta infestation conditions (Figures 3, 5). 


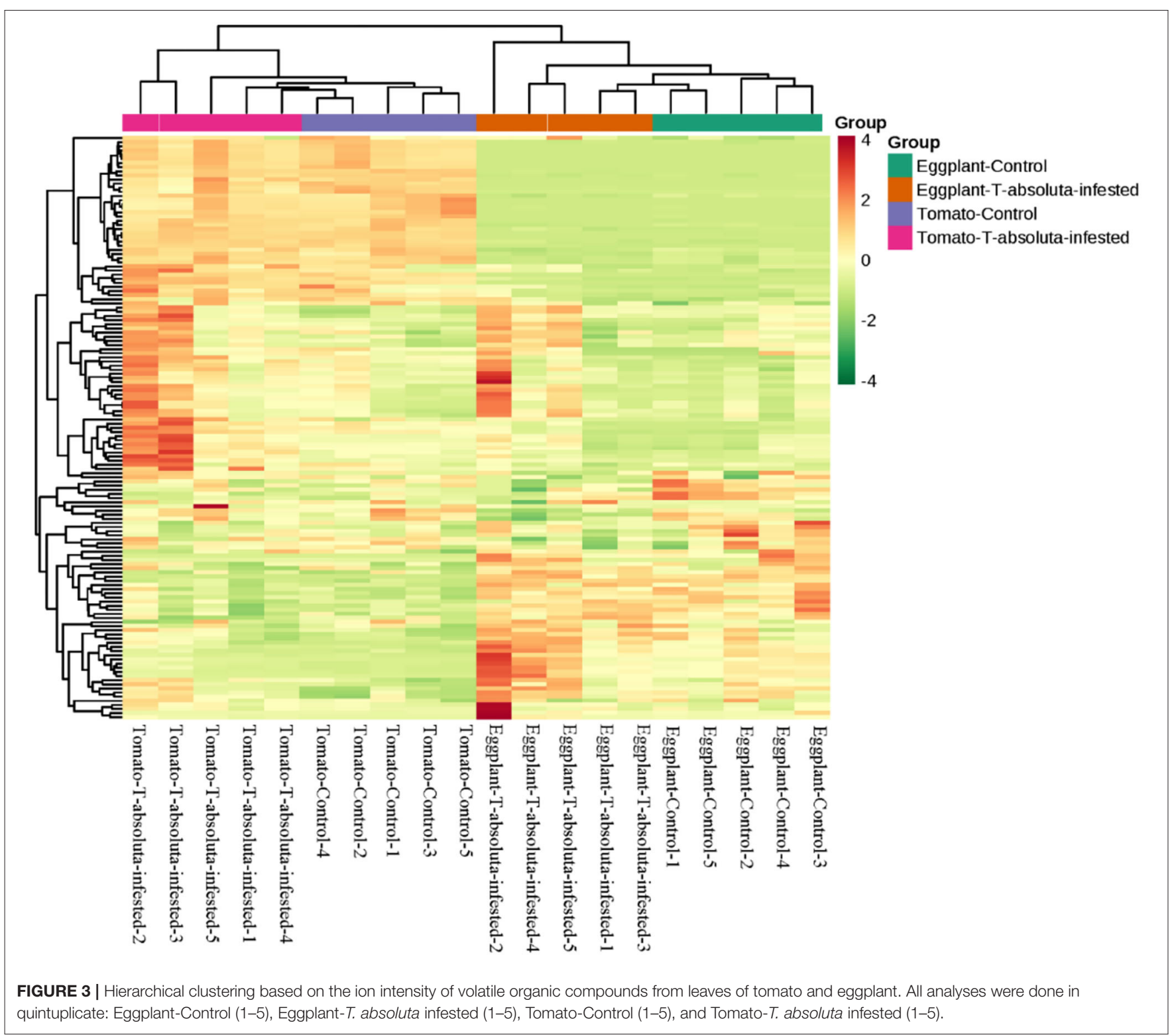

\section{Differentially Accumulated Volatile Compounds Identified in the Pairwise Groups}

We further applied a discriminant analysis of orthogonal partial least squares (OPLS-DA) at a threshold of $\log 2 \mathrm{FC}$ $\geq 1$ and variable importance in projection (VIP) $\geq 1$ to identify differentially accumulated volatile organic compounds (DAVOCs) and differentially accumulated metabolites (DAMs) among the four groups in pairwise comparisons. Overall, 96 unique DAVOCs were detected among Eggplant-Control vs. Tomato-Control, Eggplant-Control vs. Eggplant-T. absoluta infested, and Tomato-Control vs. Tomato-T. absoluta infested from 16 classes of compounds (Supplementary Tables 1-3). The extent of the occurrence and accumulation of these DAVOCs varied among the four treatments (Supplementary Figures 1A-D). There were 76 VOCs detected between Eggplant-Control and Tomato-Control; of these, 21 VOCs accumulated lower in Eggplant-Control and 55 VOCs accumulated higher in Tomato-Control (Supplementary Table 1A). This indicated that most of the VOCs found in the tomato headspace were more abundant than in eggplants (Figure 6A). For instance, 1 alkane (1,4,7,-Cycloundecatriene, 1,5,9,9-tetramethyl-, Z,Z,Z), 1 ester [2-Butenoic acid, 3-hexenyl ester, (E,Z)], 2 heterocyclic compounds [2,2'-Isopropylidenebis(5-methylfuran) and Pyrazi ne, Furfuryl Methyl Disulfide], and 10 terpenes [Nerolidol 2; gamma.-Murolene; 6-Isopropyl-1,4-dimethylnaphthalen; 10,10-Dimethyl-2,6-dimethylenebicyclo[7.2.0] undecane; 3a,7-M ethano-3aH-cyclopentacyclooctene, 1,4,5,6,7,8,9,9a-octahydro1,1,7-trimethyl-, [3aR-(3a.alpha.,7.alpha.,9a.beta.)]; alpha.Cuprenene; $\alpha$-thujene; (3E,7E)-4,8,12-Trimethyltrideca-1,3,7,11- 


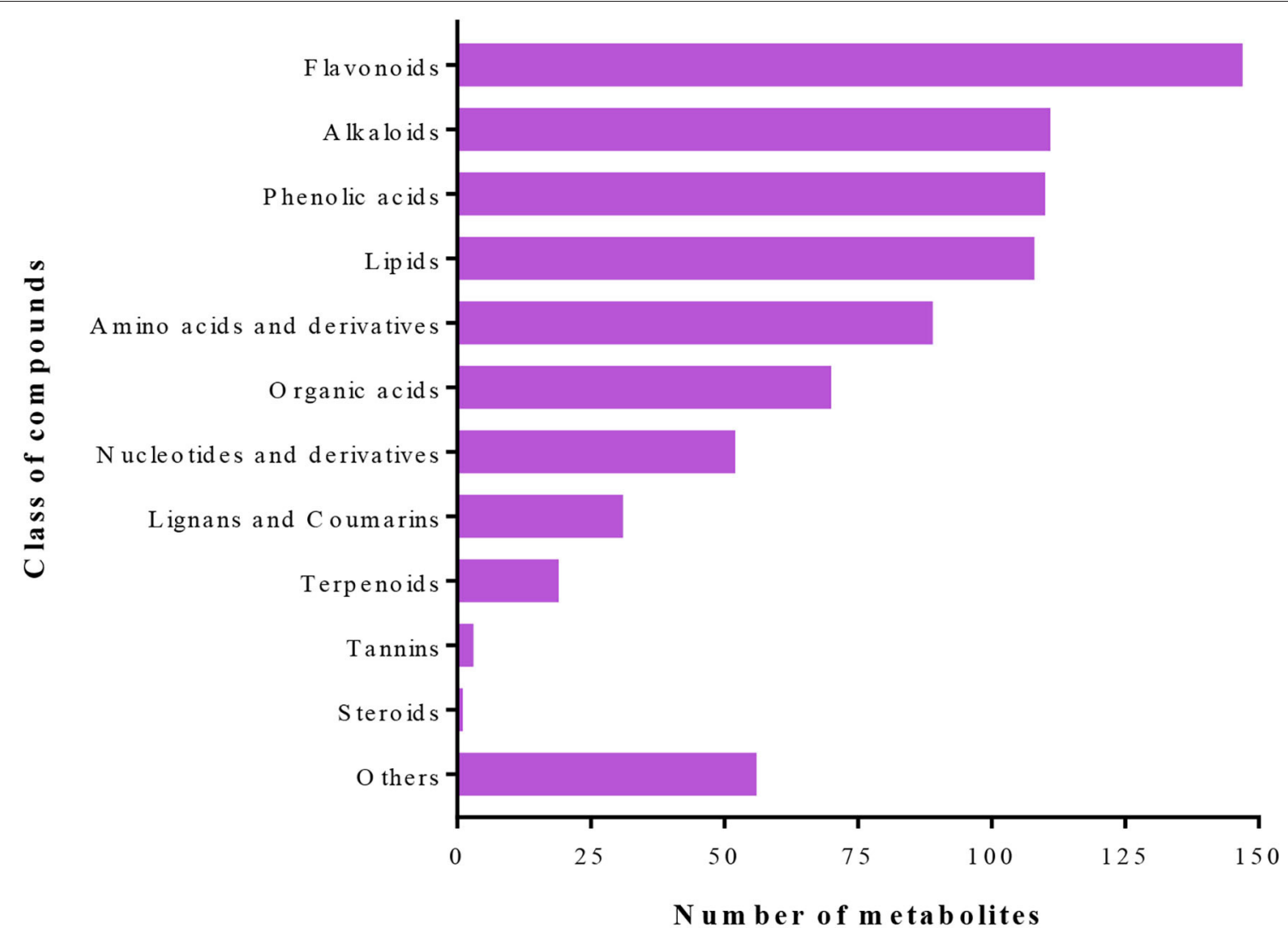

FIGURE 4 | Class of primary/secondary metabolites detected in the leaves of control and infested tomatoes and eggplants.

tetraene; (+)-4-Carene and Cyclohexene, 4-ethenyl-4-methyl3-(1-methylethenyl)-1-(1-methylethyl)-, (3R-trans)] were more abundant in uninfected tomatoes (Supplementary Table 1). The increased volatiles in tomatoes might contribute to the T. absoluta preference for tomatoes over eggplants. On the other hand, some volatiles, such as trans,cis-2,6-non-adienal, trans,trans-2,4-non-adienal, hexadecane, $\gamma$-caprolactone, and 2,2,4,4,6,8,8-heptamethylnonane, were more abundant in uninfected eggplants, and these volatiles might have repellent activities to T. absoluta. To identify volatile compounds which may act as stimulants for host location and damage by $T$. absoluta on tomatoes, we compared the volatiles found in Tomato-Control with those found in T. absoluta-infested tomato. A total of 24 DAVOCs, comprising 12 each for decreased or increased in abundance (Figure 6A), were identified. From these, four terpenes (Nerolidol 2; beta-Cyclocitral; 1,3-Cycl ohexadiene-1-carboxaldehyde, 2,6,6-trimethyl, and beta.-isoMethyl ionone), one ketone (Cyclohexanone, 2,2,6-trimethyl), one heterocyclic compound (Furan, 2-pentyl), two ester [2-Butenoic acid, 3-hexenyl ester, (E,Z) and (E)-Hex-3-enyl (E)-2-methylbut-2-enoate], two aldehydes (Benzaldehyde and 2-Ethylbenzaldehyde), and two alcohols (Cyclohexanol, 2,6dimethyl, and 1-Octen-3-ol) could be potential attractants and/or stimulants of T. absoluta in tomatoes (Supplementary Table 3).
We further compared Eggplant-Control and T. absoluta infested eggplants. There were 27 volatiles that increased in abundance upon infestation (Figure 6A). Of these, one Terpene (Nerolidol 2), one Olefin (1,3-Cyclopentadiene, 5,5-dimethyl1,2-Dipropyl), and one ester [2-Butenoic acid, 3-hexenyl ester, (E,Z)] were detected upon infestation (Supplementary Table 2). In addition, 25 volatiles accumulated 1.21-47.55 times higher in Eggplant-T. absoluta infested than Eggplant-Control (Supplementary Table 2). On the contrary, three alcohols (1-Heptanol, 1-Nonanol, and 1-Nonanol), one ester (Formic acid, octyl ester), one Olefin (1-Undecene, 9-methyl), and one Ketone (3-Nonen-5-one) accumulated 1.36-1.69 times higher in Eggplant-Control than Eggplant-T. absoluta infested (Supplementary Table 2). These volatiles may be antifeedants for T. absoluta in eggplants.

\section{Differentially Accumulated Primary/Secondary Metabolites Identified in the Pairwise Groups}

There were 442 primary/secondary metabolites found to be accumulated differentially between Eggplant-Control and Tomato-Control (Figure 6B; Supplementary Table 4). These were mostly composed of flavonoids, alkaloids, phenolic 


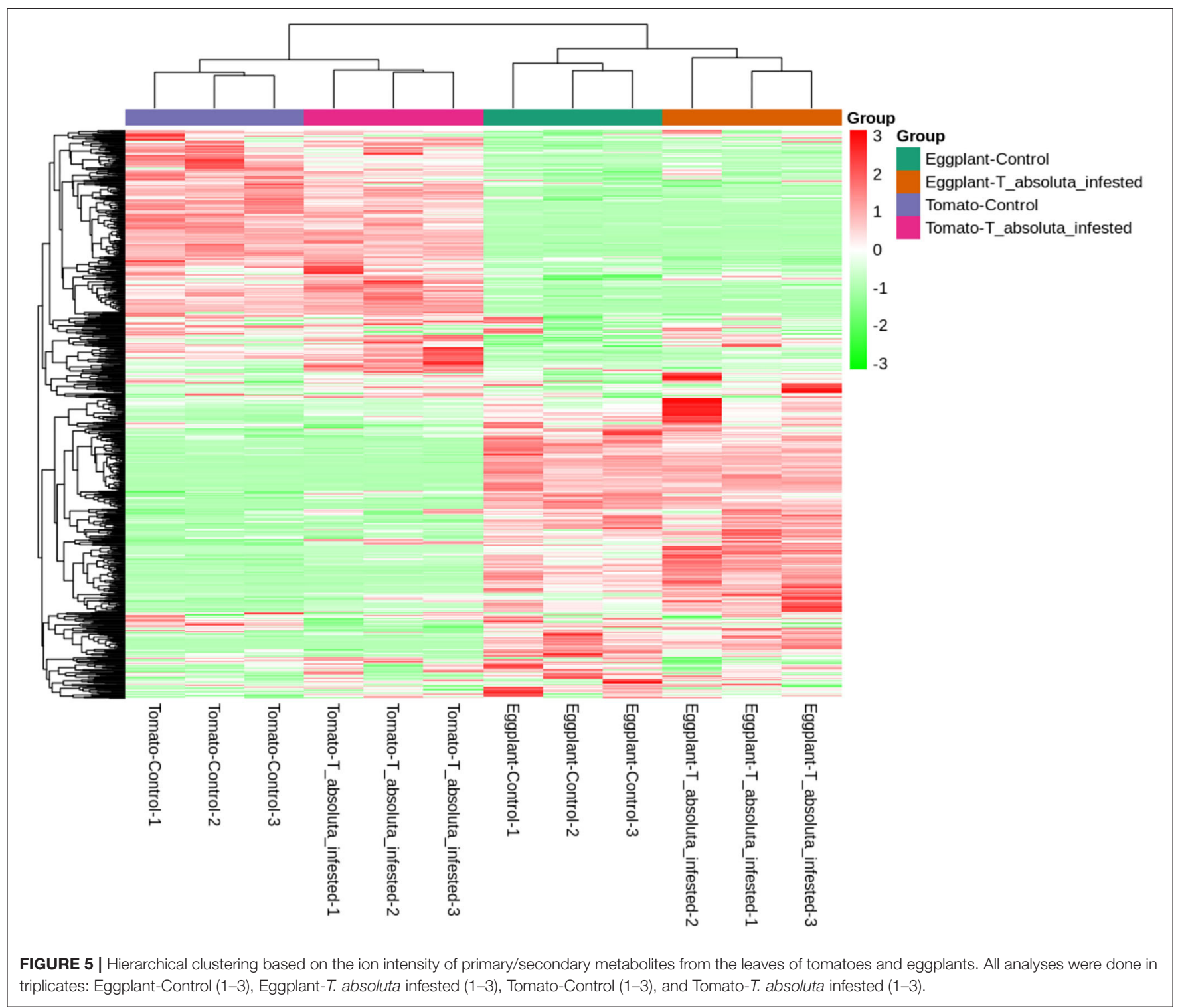

acids, and lipids (Supplementary Table 4). Additionally, these compounds mostly accumulated higher in EggplantControl than in Tomato-Control. For example, 55 alkaloids, 72 flavonoids, 15 lignans and coumarins, 55 lipids, 36 phenolic acids, 12 organic acids, 11 nucleotides and derivatives, 10 amino acids and derivatives, 2 terpenoids, and 6 other compounds were highly accumulated in Eggplant-Control or were completely absent in Tomato-Control (Supplementary Table 4). It is worth mentioning that the presence of one steroid compound, namely, "Aculeatiside A," in Eggplant-Control, which was completely absent in Tomato-Control, was found. This steroid compound may be a repellent or an antifeedant and antidigestive to $T$. absoluta in eggplants compared with tomatoes. Conversely, 205 compounds accumulated higher in Tomato-Control or were completely absent in Eggplant-Control. Among these, alkaloids (dehydrocommersonine, $\beta 1$-Tomatine, Lycoperoside $\mathrm{A}$, and Esculeogenin A-27-O-rhamnoside), amino acids and derivatives
(S-(Methyl)glutathione), Cyclo (Phe-Glu), O-Phospho-L-serine and N-Acetyl-L-Tryptophan, flavonoids (Tricin-5-O-Glucoside, Sieboldin, Quercetin-3-O-(2"-O-arabinosyl), rutinoside and Cyanidin-3-O-(6"-O-p-Coumaroyl)glucoside), lignans and Coumarins [Fraxetin (7,8-Dihydroxy-6-methoxycoumarin), 7-Methoxycoumarin, Daphnetin, and Olivil-4'-O-glucoside], organic acids (2-Hydroxyphenylacetic acid, abscisic acid, muconic acid, and (-)-Jasmonoyl-L-Isoleucine), phenolic acids (rhododendron; 3,4-Dihydroxybenzeneacetic acid; homogentisic acid; oleoside 11-methyl ester) were highly accumulated in Tomato-Control or were completely absent in Eggplant-Control (Supplementary Table 4). These compounds may be the basis for the higher preference of T. absoluta for tomatoes. These results suggested that eggplants induced several primary/secondary metabolites to enhance their defense against T. absoluta or repel T. absoluta from damaging the leaves. The absence or presence of some metabolites in the Tomato-Control compared with the 


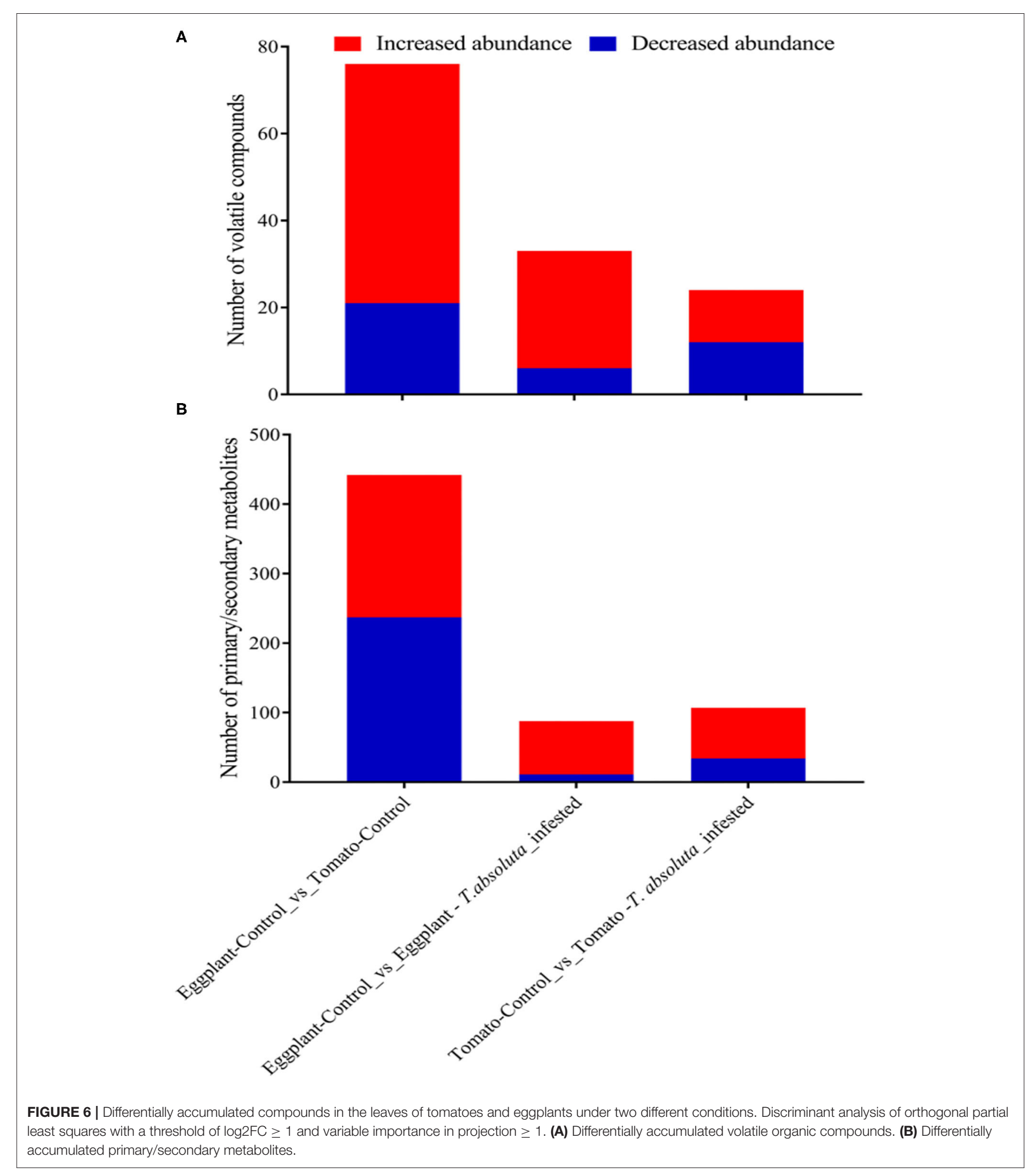

Eggplant-Control are likely to make tomatoes more prone to $T$. absoluta infestation than eggplant.

Eggplant-Control in relation to T. absoluta-infested eggplants had 88 primary/secondary metabolites with differential accumulation comprising 11 decreased and 77 increased metabolites in abundance upon infestation, respectively (Figure 6B; Supplementary Table 5). This implied that the increased abundance of several metabolites in eggplants was 
mainly to ward off or prevent the survival and multiplication of T. absoluta. Eight of such compounds exclusively detected upon infestation included two alkaloids [N-(4-O-(Glucosyl)E-feruloyl)-tyramine and esculeogenin A-27-O-rhamnoside], two amino acids and derivatives [O-Phospho-L-serine and S-(Methyl)glutathione], two flavonoids [6-Hydroxykaempferol3-O-rutin-6-O-glucoside and Quercetin-3-O-apiosyl $\left(\begin{array}{ll}\rightarrow & 2\end{array}\right)$ galactoside], one lignans and coumarins (7-Methoxycoumarin), and one organic acid (Methyl jasmonate). In contrast to these, one flavonoid (Genistein-7-O-galactoside) and one nucleotide and derivative (2-Aminopurine) were detected exclusively in Tomato-Control (Supplementary Table 5).

By comparing the metabolome of Tomato-Control with $T$. absoluta-infested tomato, a total of 107 primary/secondary metabolites that were differentially accumulated (34 downregulated and 73 upregulated) were identified (Figure 6B; Supplementary Table 6). This implied that T. absoluta-infested tomatoes induce primary/secondary metabolites that contribute to the survival and multiplication of $T$. absoluta. Eight of such compounds exclusively detected upon infestation include two alkaloids [3,5-Bis(3-methoxy-4-hydroxyphenyl)-2,3dihydro-2 $(1 \mathrm{H})$-pyridinone and Lycibarbarspermidine I], two flavonoids (Peonidin-3-O-glucoside and 6"-O-Malonylgenistin), two lignans and coumarins (Lariciresinol-4'-O-glucoside and Syringaresinol), one lipid (LysoPE 20:5), and one organic acid (2-Aminoethanesulfonic acid). In contrast to these, five flavonoids [Apigenin-6-C-rhamnoside, Patuletin7-O-rutinoside, Eriodictyol-7-O-glucoside, Delphinidin3-O-(2"'-O-p-coumaroyl) rutinoside-7-O-glucoside and Cyanidin-3-O-glucoside (Kuromanin)], two terpenoids [Betulinic acid and Medicagenic acid-3-O-glucosyl-(1,6)glucosyl-(1,3)-glucoside], one phenolic acid (Isochlorogenic acid C), one organic acid (2-Aminoethanesulfinic acid), and one amino acid and derivative ( $\gamma$-Glu-Cys) were detected exclusively in Tomato-Control (Supplementary Table 6). These compounds may have contributed to the preference of $T$. absoluta for tomatoes.

\section{Electroantennographic Response of $T$. absoluta Females to VOCs}

To verify whether the VOCs identified in this study could influence T. absoluta behavior, the electroantennogram (EAG) response of T. absoluta to 35 VOCs was investigated. The results showed that all 35 compounds could elicit electrophysiological responses in the antennae of female T. absoluta (Figure 7). Of note, 2-Methoxyestradiol, Isoamyl acetate, Geranyl Acetone, Hexyl acetate, Trans, cis-2,6-Non-adienal, $\gamma$-Caprolactone, 1Octen-3-ol, 1-Heptanol, and (Z)-2-Penten-1-ol elicited the highest EAG responses among all ligands in females.

\section{DISCUSSION}

It is well documented that T. absoluta attacks over 26 different host plant species with much preference for solanaceous species, with tomato as one of the most preferred hosts (Figure 1) (Özgökçe et al., 2016; Wellington et al., 2019; Dong and Lin,
2021). However, knowledge on the basis for this preference is limited since most of the previous studies profiled only VOCs (Anastasaki et al., 2018; Parasitoids et al., 2019) or primary/secondary metabolites (de Falco et al., 2019). Combining VOCs and primary/secondary metabolites in this present study offered the potential for identifying compounds that may have been lost/altered during domestication and breeding efforts (Alseekh et al., 2021), which may be responsible for the differential response between the two solanaceous species for the possible development of eco-friendly management strategies against T. absoluta in tomatoes.

Plants produce several compounds, both VOCs and primary/secondary metabolites, in response to insect/pest attacks (Bruce et al., 2005). These compounds act as repellents, deterrents, attractants, antinutrients, and anti-digestives either before/after attack or both. Terpenoids/terpenes and other VOCs released from plants in response to insect attacks allow parasitoids and predators to differentiate between infested and non-infested plants by assisting in the location of a host or prey (Paré P. W., 2008). In this study, 141 VOCs and 797 primary/secondary metabolites have been identified, which were largely flavonoids, alkaloids, phenolic acids, lipids, amino acids and derivatives, organic acids, nucleotides and derivatives, lignans and coumarins, terpenoids, tannins, steroids, and several other minor classes of compounds (Figures 2, 4).

We observed that eggplants emitted several compounds that were lower or completely absent in tomatoes either under control or T. absoluta-infestation conditions and vice versa (Supplementary Tables 1-6). It has been reported that the cytosolic mevalonate pathway is key in defense against insects, which starts with farnesyl diphosphate (FPP) but remains the same in insects and plants. Insects, through FPP, form a juvenile hormone that plays a role in the regulation of molting (Dubey, 2013). Steroids and sterols are products of terpenoid precursors, which are lacking in insects but can only be utilized by extracting the needed precursors for cholesterol and other steroids from their food (Belles and Martin, 2005). Therefore, the only steroid compound, namely, Aculeatiside A, potentially makes eggplant unattractive to T. absoluta (Figure 4; Supplementary Tables 1-3). A study by Bawin et al. (2014) revealed that the attraction of $T$. absoluta is mediated by the volatile signature of the host plant. Tomato leaf odors usually include volatile terpenoid compounds (Proffit et al., 2011; Anastasaki et al., 2018), which may have an influence on the preference of $T$. absoluta to tomatoes than eggplants. These observations demonstrated the essential role of plant volatiles in T. absoluta host-finding behavior. For instance, nerolidol and other compounds have been reported by earlier researchers to be present in tomatoes or induced upon infestation by T. absoluta (Supplementary Tables 1-3) (Silva et al., 2018). Most of these compounds have been demonstrated to repel the tomato borer, T. absoluta (Campolo et al., 2017).

Intriguingly, several VOCs including four terpenes (nerolidol 2; beta-Cyclocitral; 1,3-cyclohexadiene-1-carboxaldehyde, 2,6,6-trimethyl, and beta.-iso-methyl ionone), one ketone (cyclohexanone, 2,2,6-trimethyl), one heterocyclic compound (furan, 2-pentyl), two ester [2-butenoic acid, 3-hexenyl ester, 

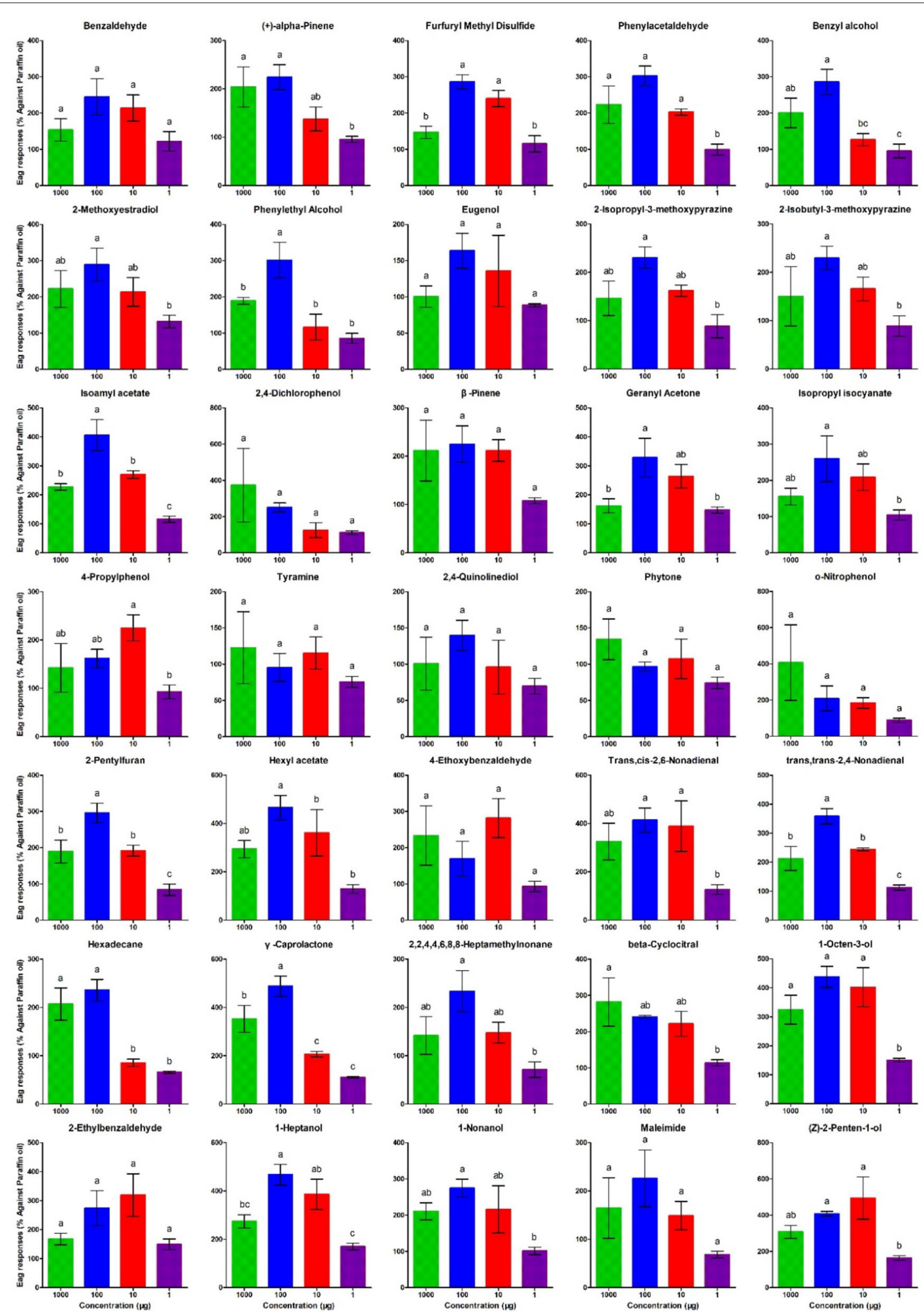

FIGURE 7 | Electroantennographic (EAG) response of $T$. absoluta females to volatile compounds. The compound names are displayed on top of each chart. The bar represents the mean of the triplicate reading from the EAG experiment. Error bars represent the SEM ( \pm SEM). Bars for each compound with a common alphabet displayed on top indicate the significant difference with Turkey's Highest Significant Difference at $P<0.05$. 
(E,Z) and (E)-hex-3-enyl (E)-2-methylbut-2-enoate], two aldehydes (benzaldehyde and 2-Ethylbenzaldehyde), and two alcohols (cyclohexanol, 2,6-dimethyl and 1-octen-3-ol) were naturally emitted by tomatoes, which may serve as attractants and stimulants to T. absoluta (Supplementary Table 1). These compounds may have contributed to the T. absoluta preference for tomato and thus, could be exploited in the pull-and-push strategy of pest management in solanaceous species (Cook et al., 2007).

Conversely, three DAVOCs were emitted by eggplants upon infestation by T. absoluta, which were nerolidol 2 (terpene), 1,3-cyclopentadiene, 5,5-dimethyl-1,2-dipropyl (olefin), and 2-butenoic acid, 3-hexenyl ester (E,Z) (ester) (Supplementary Table 2). These compounds have been demonstrated to be induced upon wounding or infestation in some plants and may contribute to repel T. absoluta survival, multiplication, and damage in eggplants. For instance, 1,4,7, cyclododecatriene, 1,5,9,9-tetramethyl was identified to have antimicrobial activity.

Among the primary/secondary metabolites differentially detected in this study, terpene compounds (Supplementary Tables 4-6; Supplementary Figures 2A,D) were in higher abundance in tomatoes than eggplants (either control or T. absoluta-infested). The abundance of terpene compounds in tomatoes are in consonance, which reported that most of these compounds are used by insect pests to recognize the host plants as attractants and feeding stimulants. Three typical examples, namely, capsianoside IV, capsianoside II, and 6'-O-D-glucosylsweroside, may serve as attractants aiding $T$. absoluta larvae to identify a susceptible host (Robert et al., 2012, 2013)

Strikingly, two flavonoid compounds [6-hydroxykaempferol3-O-rutin-6-O-glucoside and quercetin-3-O-apiosyl $\left(\begin{array}{ll}1 \rightarrow 2 & \text { 2 }\end{array}\right.$ galactoside] and other classes of compounds were induced in eggplants upon T. absoluta infestation. These flavonoid compounds have been reported to have negative effects on insects and pathogens in several crops (Simmonds, 2001). Plants emit several chemical compounds both to deter and attract insects; in some cases, the natural predators of herbivores. Among them, flavonoids have been documented to modulate oviposition and feeding. In this study, naringenin, quercetin-3-O-rutinoside, and other flavonoids have been found to be accumulated higher in eggplants or were completely absent in tomatoes. These compounds have been shown to prevent insects from laying eggs and deter Pieris rapae. Apigenin-6-C-rhamnoside, a flavonoid compound, was detected only in Tomato-Control. This compound has been reported to alter the palatability of plants to pathogens (Simmonds and Stevenson, 2001). On the contrary, this compound may work in the opposite direction compared with earlier reports (Simmonds and Stevenson, 2001). Therefore, the occurrence and abundance of flavonoid compounds under control and T. absoluta-infested Eggplant_vs_Tomato may have influenced the T. absoluta preference for tomato as a host.

The results of the EAG experiments indicated that the antennae of T. absoluta respond to all VOCs (Figure 7). This experiment suggested that a minute quantity of VOCs can act as an attractant or repellent to T. absoluta to either tomatoes or eggplants. These compounds have been reported in previous studies to repel or attract $T$. absoluta in tomatoes/eggplants (Bawin et al., 2014; Anastasaki et al., 2018). Thus, these compounds detected by the EAG experiment may contribute to the altering of the T. absoluta preference for host plants.

\section{CONCLUSIONS}

The suitability of tomatoes as hosts for T. absoluta invasion is much higher than that for eggplants based on the results of this study, which compared the metabolomes and volatilomes from both plants. The key metabolites and volatiles that are vital in modulating T. absoluta damage in tomatoes have also been reported, and future research could focus on them. These compounds could be validated for use as selection signatures in screening tomato accessions against $T$. absoluta in our future studies. The results provided in this study provide a foundation that will allow us to develop effective biological control methods of T. absoluta in cultivated tomatoes and other Solanum crops.

\section{METHODS}

\section{Plant Materials and Growth Conditions}

The tomato variety Zhefen 202 and eggplant variety Zheqie No.1 were used in this study. The plant materials were obtained from the Zhejiang Academy of Agricultural Science, and no permissions are necessary to work on such materials. Using tomato as the host plant, a population of the South American tomato pinworm, T. absoluta (Meyrick), was collected in Ili, Xinjiang (China) in 2018 and maintained continuously in an artificial climate chamber $\left[25^{\circ} \mathrm{C}\right.$, relative humidity $(60 \%)$, $16 \mathrm{~h}$ light (L): $8 \mathrm{~h}$ dark (D)]. The plants were grown in an insect-free greenhouse at the Zhejiang Academy of Agricultural Science research station (day/night temperature $24 / 20^{\circ} \mathrm{C}$, relative humidity 60\%, 16 L: 8 D) under controlled conditions. Following the cleaning and germination of tomato and eggplant seeds, the seedlings were cultivated in coconut bran to produce fruits. When the seedlings reached the two-leaf stage, they were transplanted into a plastic flowerpot with a diameter of $10 \mathrm{~cm}$ and a height of $9 \mathrm{~cm}$, where they were allowed to continue to grow. Each pot included a single plant, which was watered once every 2 days during the growing season. For each pot of host plants, $2 \mathrm{~g}$ of an OMEX 18-18-18 (OMEX, UK) macro-elements water-soluble fertilizer (containing 18 macroelements) were administered to encourage the growth of the host plants. The host plants were not exposed to any pesticides during the experiment. Furthermore, T. absoluta larvae were inoculated into eggplants at the five-leaf stage and tomato plants with 20-50 normal leaves, which were both grown in a greenhouse.

\section{Preparation of Samples for Metabolite Extraction}

Using a mixer mill MM400 with zirconia beads (Retsch $\mathrm{GmbH}$, Germany) with a $15-\mathrm{mm}$ size and running at $30 \mathrm{~Hz}$ for $1.5 \mathrm{~min}$, $3 \mathrm{~g}$ of each of the samples from the four treatments (TomatoControl, Tomato-T. absoluta infested, Eggplant-Control, and 
Eggplant-T. absoluta infested) with three biological replicates (thus, 12 samples) were crushed into powder after vacuum freeze-drying Retsch $\mathrm{GmbH}$, Germany. For the extraction of the metabolites, a 100-mg sample of the powder was further extracted overnight at $4^{\circ} \mathrm{C}$ in $1 \mathrm{ml}$ of $70 \%$ aqueous methanol. After $10 \mathrm{~min}$ of centrifugation at $10,000 \times \mathrm{g}$, the extract was removed. The extraction procedure was done, and the extracts were transferred to a fresh tube for LC-MS analysis. In order to validate the data, quality control (QC) analyses (mixing extracts for insertion into every three samples) were done. MetWare Biotechnology Company completed the analysis in accordance with previous protocols (Wang et al., 2019).

\section{Identification and Quantitation of Metabolites}

Metabolite profiling was undertaken with the use of a selfbuilt database (http://www.metware.cn) developed by MetWare Biotechnology Co. Ltd. (Wuhan, China) (Li et al., 2013; Melvin et al., 2015). With the help of secondary spectrum information, the metabolites were qualitatively evaluated. Besides, metabolite quantification was carried out utilizing the triple quadruplebar mass spectrometry in the multi-reaction monitoring (MRM) mode. A principal components analysis (PCA) plot was used to visualize the differences and similarities between and among samples. Using partial least squares-discriminant analysis (PLSDA) with a $\log 2$ FC 1 threshold and VIP 1 , we were able to identify the DAMs. An analysis of the function of DAMs was carried out in the Kyoto Encyclopedia of Genes and Genomes (KEGG) database (Kanehisa et al., 2016).

\section{Determination of Host Plant Volatiles by GC-MS}

Five healthy and insect-induced host plants (tomato and eggplant) were selected. After removing the veins, samples were lyophilized in liquid nitrogen (LN) and stored in a $-80^{\circ} \mathrm{C}$ refrigerator. Samples were later pulverized in LN and vortexed to mix evenly. The samples were put in a headspace bottle with fully automatic HS-SPME (Lee et al., 2007). The GCMS was used to identify terpenoids, benzene ring types and phenylpropanoids, fatty acid derivatives, and other volatiles. The volatile content was determined by the headspace collection method or extraction method. The SPME readings were taken at a $250^{\circ} \mathrm{C}$ aging temperature; 5-min aging time; $60^{\circ} \mathrm{C}$ heating temperature; 10 -min heating time; 20 -min adsorption time; 5-min desorption time; 5-min aging time after sample injection. The original data file obtained by GC-MS analysis was first extracted using the MassHunter software (Agilent, CA, USA).

\section{Metabolites Determination}

Extracts from the samples were analyzed using an LC-ESIMS/MS system (HPLC, Shim-pack UFLC SHIMADZU CBM30A system, Kyoto, Japan; MS, Applied Biosystems 6,500 Q TRAP, San Diego, CA, USA). It was possible to link the HPLC effluent to an electrospray ionization (ESI)-triple quadrupolelinear ion trap-mass spectrometry/mass spectrometry apparatus instead (Ap-plied Biosystems 4,500 Q TRAP, San Diego, CA, USA). The analytical conditions were modified from Wang et al. (2010) and Hsu et al. (2017). Using multiple-reaction monitoring (MRM) (Hsu et al., 2017) and the MetWare MWDB database, the researchers were able to quantify metabolites using their usual metabolic operating methods (Chen et al., 2009, 2013).

\section{Electrophysiological Responses of Tuta absoluta to DAVOCs}

There were 35 DAVOCs identified between treatments, which were selected for electroantennographic tests, including 16 increased VOCs (benzyl alcohol, 2-methoxyestradiol, phenylethyl alcohol, eugenol, 2-isopropyl-3-methoxypyrazine, 2-isobutyl-3-methoxypyrazine, isoamyl acetate, 2,4dichlorophenol, $\beta$-pinene, geranyl acetone, isopropyl isocyanate, 4-propylphenol, tyramine, 2,4-quinolinediol, phytone, and o-nitrophenol) and five decreased VOCs (trans,cis2,6-non-adienal, trans,trans-2,4-non-adienal, hexadecane, $\gamma$-caprolactone, and 2,2,4,4,6,8,8-heptamethylnonane) of tomato compared with eggplant; five increased VOCs (benzaldehyde, 2-pentylfuran, beta-cyclocitral, 1-octen3-ol, and 2-Ethylbenzaldehyde) and three decreased VOCs [(+)-alpha-pinene, 1-heptanol and 1-nonanol] of tomato after infestation; six increased VOCs (furfuryl methyl disulfide, phenylacetaldehyde, hexyl acetate, 4ethoxybenzaldehyde, maleimide, and (Z)-2-penten-1-ol) and two decreased VOCs (1-heptanol and 1-nonanol) of eggplant after infestation.

The volatiles were tested for their antennal responses by T. absoluta, which was detected utilizing the EAG detection system (Stimulus Air Controller CS-55 and SYNTECH IDIC2; Syntech, Hilversum, the Netherlands). Using three different concentrations $(1,10,100$, and $1,000 \mathrm{~g} / \mathrm{ml})$ of the standard chemicals, $10 \mathrm{ml}$ of each were applied to a filter paper strip for analysis. After allowing the solvent to evaporate for $2 \mathrm{~min}$, the strip was placed in a Pasteur pipette, and the process was repeated. Similarly, to the case of indole, the control stimulus was paraffin oil $(10 \mathrm{~m})$. The application of 2-s puffs of air through a Pasteur pipette containing the filter paper holding the stimuli resulted in test stimulations being performed. During the test, puffs of the test stimuli were administered at 1-min intervals randomly. Antennal preparations were monitored using puffs of paraffin oil administered at the start and end of each experiment and between groups of five to six compounds to ensure that they were in good condition. The antennal responses of at least three females to varying doses of substances were recorded in the laboratory. When compared with the control, the EAG reactions were normalized and reported as a percentage of the response to paraffin oil.

\section{Data Analyses}

A QC analysis was undertaken to ensure that the data was reliable before proceeding with the overall analyses. The QC sample was created by combining sample extracts for insertion into every three samples to monitor changes in the results of several analyses. The analyses of the samples were performed using the 
Analyst software (version 1.6.1; AB Sciex, Canada), which loaded data matrices with the intensity of the metabolite characteristics from the samples. The PLS-DA was used to maximize the differences in the metabolomes between the two sample pairs in this study. The relative relevance of each metabolite to the PLS-DA model was determined by utilizing the VIP as a parameter to test the hypothesis. Metabolites with a VIP $\geq 1$ and fold change $\geq 2$ or fold change $\leq 0.5$ were considered as differential metabolites for group discrimination (Chong et al., 2018). The PCA and Ward's hierarchical clustering heatmap were performed using R software (version 3.3.2; www.r-project.org) (Chong and Xia, 2019). Consequently, a metabolic pathway was constructed according to KEGG (http://www.genome.jp/kegg/) (Goto, 2000). Furthermore, a pathway analysis was performed using MetaboAnalyst (http://www.metaboanalyst.ca/) based on the change in metabolite concentration compared with the corresponding controls (Chong et al., 2018). Data obtained from EAG were subjected to an ANOVA, and means separation was done by Turkey's Highest Significant Difference (version 8, GraphPad Software, San Diego, CA, USA, www.graphpad.com) at $P \leq 0.05$.

\section{DATA AVAILABILITY STATEMENT}

The original contributions presented in the study are included in the article/Supplementary Material, further inquiries can be directed to the corresponding authors.

\section{REFERENCES}

Aksoy, E., and Kovanci, O. B. (2016). Mass trapping low-density populations of Tuta absoluta with various types of traps in field-grown tomatoes. J. Plant Dis. Protect. 3:123. doi: 10.1007/s41348-016-0003-6

Alseekh, S., Scossa, F., Wen, W., Luo, J., Yan, J., Beleggia, R., et al. (2021). Domestication of crop metabolomes: desired and unintended consequences. Trends Plant Sci. 2, 1-12. doi: 10.1016/j.tplants.2021.02.005

Anastasaki, E., Drizou, F., and Milonas, P. G. (2018). Electrophysiological and oviposition responses of tuta absoluta females to herbivore-induced volatiles in tomato plants. J. Chem. Ecol. 44, 288-298. doi: 10.1007/s10886-018-0929-1

Arnó, J., Gabarra, R., Molina, P., Godfrey, K. E., Zalom, F. G., and Sathyamurthy, R. (2019). Tuta absoluta (Lepidoptera: Gelechiidae) success on common solanaceous species from california tomato production areas. Environ. Entomol. 48, 1394-1400. doi: 10.1093/ee/nvz109

Bawin, T., De Backer, L., Dujeu, D., Legrand, P., Megido, R. C., Francis, F., et al. (2014). Infestation level influences oviposition site selection in the tomato leafminer Tuta absoluta (Lepidoptera: Gelechiidae). Insects 5, 877-884. doi: 10.3390/insects5040877

Bawin, T., Dujeu, D., De Backer, L., Francis, F., and Verheggen, F. J. (2016). Ability of Tuta absoluta (Lepidoptera: Gelechiidae) to develop on alternative host plant species. Canad. Entomol. 148, 434-442. doi: 10.4039/tce.2015.59

Belles, X., and Martin, D. (2005). The mevalonate pathway and the synthesis of juvenile hormone in insects. Annu. Rev. Entomol. 50, 181-199. doi: 10.1146/annurev.ento.50.071803.130356

Biondi, A., Guedes, R. N. C., Wan, F. H., and Desneux, N. (2018). Ecology, worldwide spread, and management of the invasive south american tomato pinworm, tuta absoluta: past, present, and future. Annu. Rev. Entomol. 63, 239-258. doi: 10.1146/annurev-ento-031616-034933

Biondi, A., Zappal,à, L., di Mauro, A., Tropea Garzia, G., Russo, A., Desneux, N., et al. (2015). Can alternative host plant and prey affect phytophagy

\section{AUTHOR CONTRIBUTIONS}

LC, XL, YH, and YL: conceptualization. LC and XL: methodology and writing-original draft preparation. LC, XL, JZ, and $\mathrm{TH}$ : software. ZZ and MH: validation. LC, XL, YW, SZ, and XR: formal analysis. LC, XL, JZ, TH, JH, ZZ, MH, SZ, and XR: investigation. $\mathrm{YH}$ and $\mathrm{YL}$ : writing-review and editing, project administration, and funding acquisition. All authors have read and approved the final version of the manuscript.

\section{FUNDING}

This research was funded by the Primary Research \& Development Plan of Lishui (No. 2020ZDYF02), the National Key R\&D Program of China (No. 22017YFC1200605), Project of State Key Laboratory for Managing Biotic and Chemical Threats to the Quality and Safety of Agro-products (No. 2010DS700124ZZ2015), and the Fujian Science and Technology Special Project (No. 22017NZ0003-1-6). The funders had no role in the study design, data collection and analysis, decision to publish, or preparation of the manuscript.

\section{SUPPLEMENTARY MATERIAL}

The Supplementary Material for this article can be found online at: https://www.frontiersin.org/articles/10.3389/fpls.2021. 757230/full\#supplementary-material

and biological control by the zoophytophagous mirid Nesidiocoris tenuis? BioControl 61, 79-90. doi: 10.1007/s10526-015-9700-5

Bruce, T. J. A., Wadhams, L. J., and Woodcock, C. M. (2005). Insect host location: a volatile situation. Trends Plant Sci. 10, 269-274. doi: 10.1016/j.tplants.2005.04.003

Campolo, O., Cherif, A., Ricupero, M., Siscaro, G., Grissa-Lebdi, K., Russo, A., et al. (2017). Citrus peel essential oil nanoformulations to control the tomato borer, Tuta absoluta: chemical properties and biological activity. Sci. Rep. 7, 1-11. doi: 10.1038/s41598-017-13413-0

Campos, M. L., De Almeida, M., Rossi, M. L., Martinelli, A. P., Litholdo Junior, C. G., Figueira, A., et al. (2009). Brassinosteroids interact negatively with jasmonates in the formation of anti-herbivory traits in tomato. J. Exp. Bot. 60, 4347-4361. doi: 10.1093/jxb/erp270

Campos, M. R., Biondi, A., Adiga, A., Guedes, R. N. C., and Desneux, N. (2017). From the Western Palaearctic region to beyond: tuta absoluta 10 years after invading Europe. Journal of Pest Science 90, 787-796. doi: 10.1007/s10340-017-0867-7

Cha, D. H., Nojima, S., Hesler, S. P., Zhang, A., Linn, C. E., Roelofs, W. L., et al. (2008). Identification and field evaluation of grape shoot volatiles attractive to female grape berry moth (Paralobesia viteana). J. Chem. Ecol. 34, 1180-1189. doi: 10.1007/s10886-008-9517-0

Chen, L., Li, X., He, T., Li, P., Liu, Y., Zhou, S., et al. (2021). Comparative biochemical and transcriptome analyses in tomato and eggplant reveal their differential responses to Tuta absoluta infestation. Genomics 5:002. doi: 10.1016/j.ygeno.2021.05.002

Chen, W., Gong, L., Guo, Z., Chen, W., Gong, L., Guo, Z., et al. (2013). A novel integrated method for large-scale detection, identification, and quantification of widely targeted metabolites: application in the study of rice metabolomics. Mol. Plant 6, 1769-1780. doi: 10.1093/mp/sst080

Chen, Y., Zhang, R., Song, Y., He, J., Sun, J., Bai, J., et al. (2009). RRLCMS/MS-based metabonomics combined with in-depth analysis of metabolic 
correlation network: finding potential biomarkers for breast cancer. Analyst 134, 2003-2011. doi: 10.1039/b907243h

Cherif, A., and Verheggen, F. (2019). A review of tuta absoluta (Lepidoptera: Gelechiidae) host plants and their impact on management strategies. Biotechnol. Agron. Soc. Environ. 23, 270-278. doi: 10.25518/1780-4507.18211

Chong, J., Soufan, O., Li, C., Caraus, I., Li, S., Bourque, G., et al. (2018). MetaboAnalyst 4.0: towards more transparent and integrative metabolomics analysis. Nucleic Acids Res. 46, W486-W494. doi: 10.1093/nar/gky310

Chong, J., and Xia, J. (2019). MetaboAnalystR: an R package for flexible and reproducible analysis of metabolomics data. Bioinformatics 19:528. doi: 10.1093/bioinformatics/bty528

Cocco, A., Deliperi, S., and Delrio, G. (2013). Control of Tuta absoluta (Meyrick) (Lepidoptera: Gelechiidae) in greenhouse tomato crops using the mating disruption technique. J. Appl. Entomol. 137:1735. doi: $10.1111 /$ j.1439-0418.2012.01735.x

Comparative biochemical and transcriptome analyses in tomato and eggplant reveal their differential responses to Tuta absoluta infestation | Mendeley Available online at: https://www.mendeley.com/search/?page=1 andquery= Comparative\%20biochemical\%20and\%20transcriptome\%20analyses \%20in\%20tomato\%20and\%20eggplant\%20reveal\%20their\%20differential $\% 20$ responses $\% 20$ to $\% 20$ Tuta $\% 20$ absoluta $\% 20$ infestationandsortBy=relevance (accessed May 10, 2021).

Cook, S. M., Khan, Z. R., and Pickett, J. A. (2007). The use of push-pull strategies in integrated pest management. Annu. Rev. Entomol. 52, 375-400. doi: 10.1146/annurev.ento.52.110405.091407

De Backer, L., Megido, R. C., Fauconnier, M. L., Brostaux, Y., Francis, F., and Verheggen, F. (2015). Tuta absoluta-induced plant volatiles: attractiveness towards the generalist predator Macrolophus pygmaeus. Arthropod Plant Interact. 9, 465-476. doi: 10.1007/s11829-015-9388-6

de Falco, B., Manzo, D., Incerti, G., Garonna, A., Pietro, E. M., and Lanzotti, V. (2019). Metabolomics approach based on NMR spectroscopy and multivariate data analysis to explore the interaction between the leafminer Tuta absoluta and tomato (Solanum lycopersicum). Phytochem. Anal. 30, 556-563. doi: $10.1002 /$ pca.2850

Desneux, N., Wajnberg, E., Wyckhuys, K. A. G., Burgio, G., Arpaia, S., NarváezVasquez, C. A., et al. (2010). Biological invasion of European tomato crops by Tuta absoluta: ecology, geographic expansion and prospects for biological control. J. Pest Sci. 83, 197-215. doi: 10.1007/s10340-010-0321-6

Dong, N. Q., and Lin, H. X. (2021). Contribution of phenylpropanoid metabolism to plant development and plant-environment interactions. J. Integr. Plant Biol. 63, 180-209. doi: 10.1111/jipb.13054

Dubey, V. S., B. R. (2013). An overview of the non-mevalonate pathway for terpenoid biosynthesis in plants. J. Biosci. 28, 637-646. doi: 10.1007/BF02703339

El-Arnaouty, S. A., Pizzol, J., Galal, H. H., Kortam, M. N., Afifi, A. I., Beyssat, V., et al. (2014). Assessment of two trichogramma species for the control of tuta absoluta in North African Tomato Greenhouses 22, 801-809. doi: 10.4001/003.022.0410

Fatouros, N. E., Cusumano, A., Danchin, E. G. J., and Colazza, S. (2016). Prospects of herbivore egg-killing plant defenses for sustainable crop protection. Ecol. Evol. 6, 6906-6918. doi: 10.1002/ece3.2365

Gerszberg, A., Hnatuszko-Konka, K., Kowalczyk, T., and Kononowicz, A. K. (2015). Tomato (Solanum lycopersicum L.) in the service of biotechnology. Plant Cell Tissue Organ Cult. 120, 881-902. doi: 10.1007/s11240-014-0664-4

Goto, S. (2000). KEGG: Kyoto encyclopedia of genes and genomes. Nucleic Acids Res. 28, 27-30. doi: 10.1093/nar/28.1.27

Gripenberg, S., Mayhew, P. J., Parnell, M., and Roslin, T. (2010). A meta-analysis of preference-performance relationships in phytophagous insects. Ecol. Lett. 13, 383-393. doi: 10.1111/j.1461-0248.2009.01433.x

Guimapi, R. A., Srinivasan, R., Tonnang, H. E., Sotelo-Cardona, P., and Mohamed, S. A. (2020). Exploring the mechanisms of the spatiotemporal invasion of Tuta absoluta in Asia. Agriculture 10, 1-12. doi: 10.3390/agriculture10040124

Han, P., Bayram, Y., Shaltiel-Harpaz, L., Sohrabi, F., Saji, A., Esenali, U. T., et al. (2018). Tuta absoluta continues to disperse in Asia: damage, ongoing management and future challenges. J. Pest Sci. 92, 1317-1327. doi: 10.1007/s10340-018-1062-1

Howe, G. A., and Jander, G. (2008). Plant immunity to insect herbivores. Annu. Rev. Plant Biol. 59, 41-66. doi: 10.1146/annurev.arplant.59.032607.092825
Hsu, H. J., Huang, R. F., Kao, T. H., Inbaraj, B. S., and Chen, B. H. (2017). Preparation of carotenoid extracts and nanoemulsions from Lycium barbarum L. and their effects on growth of HT-29 colon cancer cells. Nanotechnology 28:e56. doi: 10.1088/1361-6528/aa5e86

Kanehisa, M., Sato, Y., Kawashima, M., Furumichi, M., and Tanabe, M. (2016). KEGG as a reference resource for gene and protein annotation. Nucleic Acids Res. 32, 277-280. doi: 10.1093/nar/gkh063

Kavanaugh, C. J., Trumbo, P. R., and Ellwood, K. C. (2007). The U.S. food and drug administration's evidence-based review for qualified health claims: tomatoes, lycopene, and cancer. J. Nat. Cancer Instit. 99, 1074-1085. doi: 10.1093/jnci/djm037

Langa, T. P., Dantas, K. C. T., Pereira, D. L., de Oliveira, M., Ribeiro, L. M. S., and Siqueira, H. A. A. (2021). Basis and monitoring of methoxyfenozide resistance in the South American tomato pinworm Tuta absoluta. J. Pest Sci. 2021, 1-14. doi: 10.1007/s10340-021-01378-z

Lee, J. M., Kim, D. H., Chang, P. S., and Lee, J. H. (2007). Headspace-solid phase microextraction (HS-SPME) analysis of oxidized volatiles from free fatty acids (FFA) and application for measuring hydrogen donating antioxidant activity. Food Chem. 105, 414-420. doi: 10.1016/j.foodchem.2006.12.059

Leitner, M., Boland, W., and Mithöfer, A. (2005). Direct and indirect defences induced by piercing-sucking and chewing herbivores in Medicago truncatula. New Phytol. 167, 597-606. doi: 10.1111/j.1469-8137.2005.01426.x

Li Xiaowei, L. D. W. Y. (2019). Host-plant suitability of South America tomato pinworm Tuta absoluta(Meyrick)(Lepidoptera: Gelechiidae) on four solanaceous plants. Plant Quarant. 11, 3-7.

Li, C., Yan, J. M., Li, Y. Z., Zhang, Z. C., Wang, Q. L., and Liang, Y. (2013). Silencing the SpMPK1, SpMPK2, and SpMPK3 genes in tomato reduces abscisic acid-mediated drought tolerance. Int. J. Mol. Sci. 14, 21983-21996. doi: 10.3390/ijms141121983

Li, X., Li, D., and Guo Wenchao, L. Y. (2019). Study on the adaptability of T. absoluta to four species of Solanaceae. Phytosanitary 12:3.

Li, X., wei, Li, D., Zhang, Z., jun, Huang, J., Zhang, J., ming, Hafeez, M., et al. (2021). Supercooling capacity and cold tolerance of the South American tomato pinworm, Tuta absoluta, a newly invaded pest in China. J. Pest Sci. 94, 845-858. doi: 10.1007/s10340-020-01301-y

Machekano, H., Mutamiswa, R., and Nyamukondiwa, C. (2018). Evidence of rapid spread and establishment of Tuta absoluta (Meyrick) (Lepidoptera: Gelechiidae) in semi-arid Botswana. Agric. Food Secur. 7, 1-12. doi: 10.1186/s40066-018-0201-5

Maluf, W. R., de Silva, V. F., das Cardoso, M. G., Gomes, L. A. A., Neto, Á. C. G., Maciel, G. M., et al. (2010). Resistance to the South American tomato pinworm Tuta absoluta in high acylsugar and/or high zingiberene tomato genotypes. Euphytica 176, 113-123. doi: 10.1007/s10681-010-0234-8

Mansour, R., Brévault, T., Chailleux, A., Cherif, A., Grissa-Lebdi, K., Haddi, K., et al. (2018). Occurrence, biology, natural enemies and management of Tuta absoluta in Africa. Entomol. General. 38, 83-112. doi: 10.1127/entomologia/2018/0749

Melvin, P., Prabhu, S. A., Veena, M., Shailasree, S., Petersen, M., Mundy, J., et al. (2015). The pearl millet mitogen-activated protein kinase PgMPK4 is involved in responses to downy mildew infection and in jasmonic- and salicylic acid-mediated defense. Plant Mol. Biol. 87, 287-302. doi: 10.1007/s11103-014-0276-8

Mirhosseini, M. A., Fathipour, Y., Holst, N., Soufbaf, M., and Michaud, J. P. (2019). An egg parasitoid interferes with biological control of tomato leafminer by augmentation of Nesidiocoris tenuis (Hemiptera: Miridae). Biol. Control 133, 34-40. doi: 10.1016/j.biocontrol.2019.02.009

Mutamiswa, R., Machekano, H., and Nyamukondiwa, C. (2017). First report of tomato leaf miner, Tuta absoluta (Meyrick) (Lepidoptera: Gelechiidae), in Botswana. Agric. Food Secur. 6, 1-10. doi: 10.1186/s40066-0170128-2

Negi, S., Sharma, P. L., Sharma, K. C., and Verma, S. C. (2018). Effect of host plants on developmental and population parameters of invasive leafminer, Tuta absoluta (Meyrick) (Lepidoptera: Gelechiidae). Phytoparasitica 46, 213-221. doi: 10.1007/s12600-018-0661-y

Özgökçe, M. S., Bayindir, A., and Karaca, I. (2016). Temperature-dependent development of the tomato leaf miner, Tuta absoluta (Meyrick) (Lepidoptera: Gelechiidae) on tomato plant Lycopersicon esculentum Mill. (Solanaceae). Turkiye Entomoloji Dergisi 40, 51-59. doi: 10.16970/ted.64743 
Parasitoids, E., Milonas, P. G., Anastasaki, E., and Partsinevelos, G. (2019). Oviposition-induced volatiles affect electrophysiological and behavioral responses of egg parasitoids. Insects 10:437. doi: 10.3390/insects10120437

Paré P. W., and F. M. A. (2008). "Natural Enemy Attraction to Plant Volatiles," in Encyclopedia of Entomology, ed. Capinera J.L (Berlin Heidelberg: Springer), 24-57.

Proffit, M., Birgersson, G., Bengtsson, M., Reis, R., Witzgall, P., and Lima, E. (2011). Attraction and oviposition of tuta absoluta females in response to tomato leaf volatiles. J. Chem. Ecol. 37, 565-574. doi: 10.1007/s10886-011-9961-0

Robert, C. A. M., Erb, M., Hiltpold, I., Hibbard, B. E., Gaillard, M. D. P., Bilat, J., et al. (2013). Genetically engineered maize plants reveal distinct costs and benefits of constitutive volatile emissions in the field. Plant Biotechnol. J. 11, 628-639. doi: 10.1111/pbi.12053

Robert, C. A. M., Veyrat, N., Glauser, G., Marti, G., Doyen, G. R., Villard, N., et al. (2012). A specialist root herbivore exploits defensive metabolites to locate nutritious tissues. Ecol. Lett. 15, 55-64. doi: 10.1111/j.1461-0248.2011.01708.x

Rostami, E., Madadi, H., Abbasipour, H., Allahyari, H., and Cuthbertson, A. G. S. (2020). Pest density influences on tomato pigment contents: the South American tomato pinworm scenario. Entomol. General. 40, 195-205. doi: 10.1127/entomologia/2020/0788

Sankarganesh, E., Firake, D. M., Sharma, B., Verma, V. K., and Behere, G. T. (2017). Invasion of the South American Tomato Pinworm, Tuta absoluta, in northeastern India: a new challenge and biosecurity concerns. Entomologia Generalis 36, 335-345. doi: 10.1127/entomologia,/2017/0489

Shah, S. H., Ali, S., and Jan, S. A. (2015). Piercing and incubation method of in planta transformation producing stable transgenic plants by overexpressing DREB1A gene in tomato (Solanum lycopersicum Mill.). Plant Cell Tiss Organ Cult 120, 1139-1157. doi: 10.1007/s11240-014-0670-6

Silva, G. A., Queiroz, E. A., Arcanjo, L. P., Lopes, M. C., Araújo, T. A., Galdino, T. S. V., et al. (2021). Biological performance and oviposition preference of tomato pinworm Tuta absoluta when offered a range of Solanaceous host plants. Sci. Rep. 11, 1-10. doi: 10.1038/s41598-020-80434-7

Silva, J. E., Ribeiro, L. M., da, S., Vinasco, N., Guedes, R. N. C., and Siqueira, H. Á. A. (2018). Field-evolved resistance to chlorantraniliprole in the tomato pinworm Tuta absoluta: inheritance, cross-resistance profile, and metabolism. J. Pest Sci. 92, 1421-1431. doi: 10.1007/s10340-018-1064-Z

Simmonds, M. S., and Stevenson, P. C. (2001). Effects of isoflavonoids from Cicer on larvae of Heliocoverpa armigera. J. Chem. Ecol. 27, 965-977. doi: 10.1023/A:1010339104206

Simmonds, M. S. J. (2001). Importance of flavonoids in insect-plant interactions: Feeding and oviposition. Phytochem 56, 245-252. doi: 10.1016/S0031-9422(00)00453-2

Sylla, S., Brévault, T., Monticelli, L. S., Diarra, K., and Desneux, N. (2019). Geographic variation of host preference by the invasive tomato leaf miner Tuta absoluta: implications for host range expansion. J. Pest Sci. 92, 1387-1396. doi: 10.1007/s10340-019-01094-9

Tarusikirwa, V. L., Machekano, H., Mutamiswa, R., Chidawanyika, F., and Nyamukondiwa, C. (2020). Tuta absoluta (Meyrick) (lepidoptera: Gelechiidae) on the "offensive" in Africa: Prospects for integrated management initiatives. Insects 11, 1-33. doi: 10.3390/insects11110764
Torres, J. B., Faria, C. A., Evangelista, W. S. J., and Pratissoli, D. (2001). Withinplant distribution of the leaf miner Tuta absoluta (Meyrick) immatures in processing tomatoes, with notes on plant phenology. Int. J. Pest Manag. 47, 173-178. doi: 10.1080/02670870010011091

Wang, C. C., Chang, S. C., Inbaraj, B. S., and Chen, B. H. (2010). Isolation of carotenoids, flavonoids and polysaccharides from Lycium barbarum L. and evaluation of antioxidant activity. Food Chem. 120, 184-192. doi: 10.1016/j.foodchem.2009.10.005

Wang, Y., Zeng, X., Xu, Q., Mei, X., Yuan, H., Jiabu, D., et al. (2019). Metabolite profiling in two contrasting Tibetan hulless barley cultivars revealed the core salt-responsive metabolome and key salt-tolerance biomarkers. AoB Plants 11, 1-14. doi: 10.1093/aobpla/plz021

War, A. R., Paulraj, M. G., War, M. Y., and Ignacimuthu, S. (2011). Role of salicylic acid in induction of plant defense system in chickpea (cicer arietinum 1). Plant Signal. Behav. 6, 1787-1792. doi: 10.4161/psb.6.11.17685

Wellington, N., Shanmuganathan, M., Souza, R. J., De, D.esai, D., Anand, S. S., and Britz-mckibbin, P. (2019). Metabolic trajectories following contrasting prudent and western diets from food provisions: identifying robust biomarkers of shortterm changes in habitual diet. Nutrients 11:2407. doi: 10.3390/nu11102407

Zappal,à, L., Biondi, A., Alma, A., Al-Jboory, I. J., Arn,ò, J., Bayram, A., et al. (2013). Natural enemies of the South American moth, Tuta absoluta, in Europe, North Africa and Middle East, and their potential use in pest control strategies. J. Pest Sci. 86, 635-647. doi: 10.1007/s10340-013-0531-9

Zhang, G., fen, Ma, D., ying, Wang, Y., sheng, Gao, Y., hua, Liu, W., xue, Zhang, R., et al. (2020). First report of the South American tomato leafminer, Tuta absoluta (Meyrick), in China. J. Integr. Agric. 19, 1912-1917. doi: 10.1016/S2095-3119(20)63165-3

Zhou, Z., Gao, H., Ming, J., Ding, Z., Lin, X., and Zhan, R. (2020). Combined Transcriptome and Metabolome analysis of Pitaya fruit unveiled the mechanisms underlying Peel and pulp color formation. BMC Genom. 21:734. doi: 10.1186/s12864-020-07133-5

Conflict of Interest: The authors declare that the research was conducted in the absence of any commercial or financial relationships that could be construed as a potential conflict of interest.

Publisher's Note: All claims expressed in this article are solely those of the authors and do not necessarily represent those of their affiliated organizations, or those of the publisher, the editors and the reviewers. Any product that may be evaluated in this article, or claim that may be made by its manufacturer, is not guaranteed or endorsed by the publisher.

Copyright (c) 2021 Chen, Li, Zhang, He, Huang, Zhang, Wang, Hafeez, Zhou, Ren, Hou and Lu. This is an open-access article distributed under the terms of the Creative Commons Attribution License (CC BY). The use, distribution or reproduction in other forums is permitted, provided the original author(s) and the copyright owner(s) are credited and that the original publication in this journal is cited, in accordance with accepted academic practice. No use, distribution or reproduction is permitted which does not comply with these terms. 